\section{DNA break repair goes live}

Homologous recombination (HR) is used to repair DNA doublestrand breaks (DSBs) that threaten the genomic stability of prokaryotic and eukaryotic cells. In bacteria, the RecBCD complex processes the ends of DSBs to generate 3 '-terminal single-strand extensions for RecA recruitment. RecA binding nucleates assembly of a

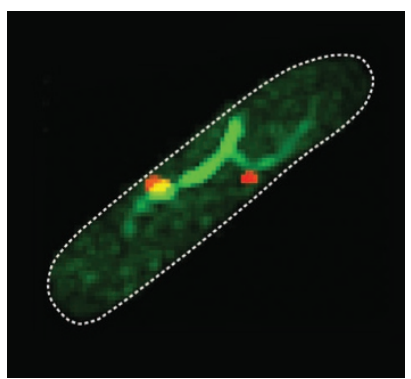
nucleoprotein filament that aligns

with sequences of a homologous duplex and promotes strand invasion leading to exchange. Although RecA function has been extensively studied in vitro, its intracellular activities have not been directly observed, and it is not known whether HR repair is restricted to sister duplexes that remain in proximity after replication. Now, Sherratt and colleagues use a combination of super-resolution structured illumination microscopy (SIM) and wide-field time-lapse microscopy to monitor the recruitment of fluorescently labeled RecA proteins to RecBCD-processed DSBs induced by I-SceI endonuclease in living cells. Remarkably, they observe pairing and recombination between homologous sister loci that have segregated to opposite halves of the cell after replication. Time-lapse analysis of repair-competent Escherichia coli revealed that both ends of the DSB are processed simultaneously by RecBCD and recruit $\sim 3,000$ RecA molecules to generate filaments termed 'RecA bundles' that extend along the long axis of the cell between the bulk DNA and the inner membrane. The cut ends then travel towards the uncut sister locus, resulting in rapid sister pairing and RecAbundle disassembly. Repair-deficient RecA mutants unable to bind or hydrolyze ATP do not form bundles, consistent with the bundles being functionally relevant. Recombination factors that promote downstream events are recruited to the sites after bundle disassembly. The current findings suggest that RecA bundles facilitate the homology search by directionally 'channeling' the motion of cut ends over long distances, by an as-yet-uncharacterized mechanism, to permit efficient HR-mediated DNA repair after sister-chromosome segregation. (Nature doi:10.1038/ nature12868, 22 December 2013)

$B M$

\section{$\mathrm{m}^{6} \mathrm{~A}$ RNA methylation in mammals}

Thousands of mRNAs contain modified $N^{6}$-methyladenosine $\left(\mathrm{m}^{6} \mathrm{~A}\right)$, but the functional significance of this RNA modification in mammals has been unknown. Zhao and colleagues now report that the putative $\mathrm{m}^{6} \mathrm{~A}$ methyltransferase Mettl3 and the related Mettl14 protein are required for $\mathrm{m}^{6} \mathrm{~A}$ formation in vitro and in vivo. The two proteins appear to function synergistically, interact and regulate each other's stability. Knockdown of Mettl3 and Mettl14 in mouse embryonic stem cells (mESCs) resulted in significantly decreased $\mathrm{m}^{6} \mathrm{~A}$ levels, and genome-wide $\mathrm{m}^{6} \mathrm{~A}$ immunoprecipitation combined with highthroughput sequencing identified $>4,700$ possible RNA substrates. The Mettl3- and Mettl14-knockdown cells showed similar phenotypes typical of lost self-renewal capability. Microarray and quantitative reverse-transcription PCR analyses indicated that most pluripotency factors were downregulated in the knockdown cells, whereas some developmental regulators were upregulated. Knockdown cells also showed increased RNA stability, whereas $\mathrm{m}^{6} \mathrm{~A}$-modified mRNAs showed accelerated decay rates. To gain insight into the underlying mechanism, the authors focused on the RNA-stabilizing protein $\mathrm{HuR}$ and noted an inverse correlation between HuR binding and $\mathrm{m}^{6} \mathrm{~A}$ modified RNA in knockdown cells. Indeed, RNA stability of an $\mathrm{m}^{6} \mathrm{~A}$ target mRNA, but not control transcripts that were not $\mathrm{m}^{6} \mathrm{~A}$ targets or lacked HuR-binding sites, was regulated in a HuR-dependent manner. HuR also increased RNA stability of Mettl3 or Mettl14 targets in knockdown cells by blocking microRNA targeting. Together, these data imply that the presence of $\mathrm{m}^{6} \mathrm{~A}$ methylation in some transcripts, particularly those encoding developmental regulators, blocks $\mathrm{HuR}$ binding and destabilizes them, thereby maintaining the mESC ground state. (Nat. Cell Biol. doi:10.1038/ncb2902, 7 January 2014) AH

\section{Hijacking through mimicry}

Viruses use a variety of strategies to evade and/ or neutralize host defense systems. In the case of HIV and other lentiviruses, the viral protein Vif has a key role in neutralizing the host restriction factor APOBEC3G, which functions to inhibit viral

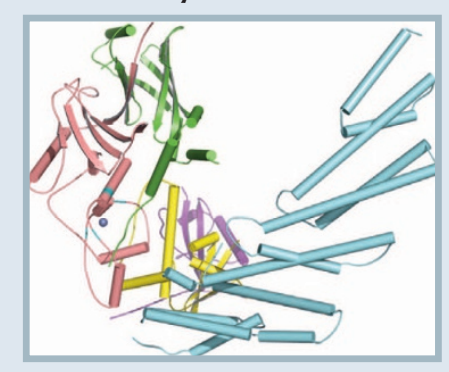

replication. Vif interaction results in targeted degradation of $A P O B E C 3 G$ through recruitment of an E3 ubiquitin ligase complex formed by CUL5 and the substrate adaptors ELOB and ELOC, in addition to the host protein CBF- $\beta$. To shed light on the precise mechanism by which Vif hijacks host factors to neutralize innate defenses, Huang and colleagues have solved the crystal structure of a pentameric HIV Vif-CBF- $\beta$-CUL5-ELOB-ELOC complex. Vif forms a new fold composed of two domains, separated by a zinc-binding site, that bear resemblance to the cellular proteins SOCS2 and VHL, both substrate-binding proteins known to interact with ELOBC. The complex, organized through interactions with Vif, assumes a U shape with CUL5 forming one arm and Vif-CBF- $\beta$ the other, while ELOC interacts with both Vif and CUL5 to form the curve of the U. ELOB makes contact with only ELOC. Structural comparison shows that Vif and SOCS2, despite limited sequence homology, use the same interface to bind ELOC. The known preference of Vif for binding CUL5 over the related E3 ligase CUL2 could be accounted for by specific residues within CUL5 that are not conserved in CUL2. Those same residues were also previously shown to mediate the specific interaction of CUL5 with SOCS2, thus further supporting the idea that Vif acts as a SOCS2 mimic to hijack cellular proteins. In addition to revealing the structural basis for Vif activity, the structure may prove useful in the design of anti-HIV drugs. (Nature 505, 229-233, 2014) $S L$ 